\section{ESTUDIO \\ ETNOARQUEOLÓGICO DE UN CAMPAMENTO TEMPORAL NDOROBO (MAASAI) EN KULALU (KENIA)}

\author{
AN ETHNOARCHAEOLOGICAL \\ STUDY OF A NDOROBO (MAASAI) \\ TEMPORARY CAMP SITE IN KULALU \\ (KENYA)
}

\section{MANUEL DOMÍNGUEZ-RODRIGO (*) RAQUEL MARTÍ LEZANA (**)}

\section{RESUMEN}

En este trabajo se presenta un estudio etnoarqueológico orientado al refinamiento de las diagnosis de las pautas de conducta antrópicas en la intervención de carcasas y en las formas de alteración de los restos por efecto de la participación posterior de agentes carnívoros carroñeros. Se pretende conocer las formas de distribución espacial de ciertas actividades humanas elementales (desarticulación de la carcasa, lugares de consumo de carne y médula ósea), los sesgos de la información introducidos por la intervención de los otros agentes y el modo en que este conocimiento puede aplicarse al discernimiento de semejantes procesos en el registro arqueológico. El objeto es analizar las improntas de unos y otros factores en la acumulación ósea final y perfilar el marco referencial en el que se deben hacer inteligibles estas cuestiones a partir de la información obtenida en yacimientos paleolíticos.

(*) Department of Anthropology. Rutgers University. Douglas Campus. New Brunswick. New Jersey 08903-0270. USA.

(***) Plaza Almuñécar, 7. 28008 Madrid.

El artículo fue remitido en su versión final el 6-VI-96.

\begin{abstract}
In this paper an ethnoarchaeological study is presented, focused on the refinement of the diagnosis of human behaviour on carcass processing and on the multiple ways of bone modification by post-ravaging carnivores. It is pretended to know the spatial distribution patterns of determined human activities (carcass processing, places of meat and marrow consumption), the biases introduced by the intervention of other nonhuman agencies, and the way that such overlapping processes can be recognised in the archaeological record. The aim is to analyse the material traces of all these factors and to improve the referential framework with which Paleolithic sites must be understood and accounted for.
\end{abstract}

Palabras clave: Etnoarqueología. Distribución espacial. Carne. Médula. Marcas de corte. Marcas de dientes. Representación esquelética.

Key words: Ethnoarchaeology. Spatial distribution. Meat. Marrow. Cut marks. Tooth marks. Skeletal representation. 


\section{INTRODUCCIÓN}

La disciplina etnoarqueológica constituye una herramienta de suma utilidad para la Arqueología, jugando un papel esencial en la comprensión de ciertos procesos conductuales antrópicos con resultados materiales concretos y diagnosis propias. Las referencias etnográficas a la hora de interpretar comportamientos humanos pretéritos a partir de registros arqueológicos son una constante que no se ha visto acompañada, en la mayor parte de las veces, por un desarrollo paralelo de estudios etnoarqueológicos. A menudo, algunos de estos estudios son descriptivos, dando la errónea imprensión de que la Etnoarqueología debe utilizarse como un referente estático, siendo en realidad todo lo contrario. En vez de constituir un campo de investigación descriptivo, su proceder debe ser analítico, implicando con semejante postura el análisis de procesos (causa-efecto) en cuya comprensión se establezcan diagnosis susceptibles de ser aplicadas al registro arqueológico, para distinguir entre formas distintas de comportamientos.

Desde esta aproximación dinámica y con el afán de profundizar en lo que Binford (1981) denominó "teoría de alcance medio", decidimos llevar a cabo un estudio etnoarqueológico orientado por un lado a intentar establecer pautas para reconstruir algunas de las formas más básicas de la conducta humana (estudio espacial de la desarticulación, preparación y consumo de un animal, y análisis de los patrones de alteración antrópica sobre los restos conservados), y por otra parte, observar el modo en que las improntas materiales generadas de esta manera se manifiestan y conservan tras un proceso posterior de modificación y consumo/dispersión llevado a cabo por los animales carroñeros (hienas y chacales). En realidad, intentamos establecer categorías diagnósticas que permitieran aproximarnos mejor a la interpretación de la conducta humana en contextos arqueológicos (como los plio-pleistocénicos) en los que la acción antrópica precede a un fuerte proceso destructivo por parte de hienas y chacales (Blumenschine, 1988; Blumenschine \& Marean, 1993; Bunn,
1982; Capaldo, 1995; Marean et alii, 1992, Domínguez-Rodrigo, 1994). Aunque una parte de este tipo de investigación de consumo humano de carcasas y alteración de carnívoros ya se había realizado de manera experimental, con estudios concretos con carnívoros en zoológicos y sabanas africanas (Blumenschine \& Marean, 1993; Marean et alii, 1992), no se había llevado a cabo reproduciendo el proceso completo de principio a fin (tal y como se supone que sucedió en buena parte del registro arqueológico) sin interrupción y sobre todos los elementos del esqueleto. Además, tampoco se había hecho sin la intervención del investigador en la modificación y disposición de los restos, que puede influir en la distorsión de dicho proceso. Los estudios etnoarqueológicos de Brain (1981) o Binford (1981) presentan el inconveniente de analizar sólo parte del proceso y con carnívoros que son perros domésticos, de menor capacidad destructiva que los hiénidos. Por otro lado, Bartram et alii (1991) realizaron un estudio considerable sobre la disposición "arqueológica" de los restos generados por poblaciones bosquimanas en sus campamentos, pero no consideraron del mismo modo que nosotros haremos en este trabajo las pautas de alteración antrópicas y carnívoras de los huesos. A este respecto, y para evitar la posible distorsión que introduce el investigador, hemos querido que fuesen poblaciones humanas acostumbradas a la manipulación de carcasas las que llevasen a cabo su explotación en sus lugares habituales, en contextos ecológicos de sabana, con una dinámica trófica similar a la que existió durante una buena parte del Plio-Pleistoceno no sólo en África, sino también en ciertas zonas de Eurasia, para poder evaluar los procesos de destrucción y conservación de restos y reconstruir a partir de éstos las conductas que los generaron.

Como punto de partida necesitábamos un yacimiento etnoarqueológico de "alta resolución", es decir, un lugar utilizado por un grupo humano, en el que el número de actividades realizadas fuese reducido o que la cantidad de estas que dejan huellas materiales fuese limitada, para poder facilitar su lectura. Durante el verano de 1995 empezamos la primera campa-

T. P., 53, n. ${ }^{\circ} 2,1996$ 


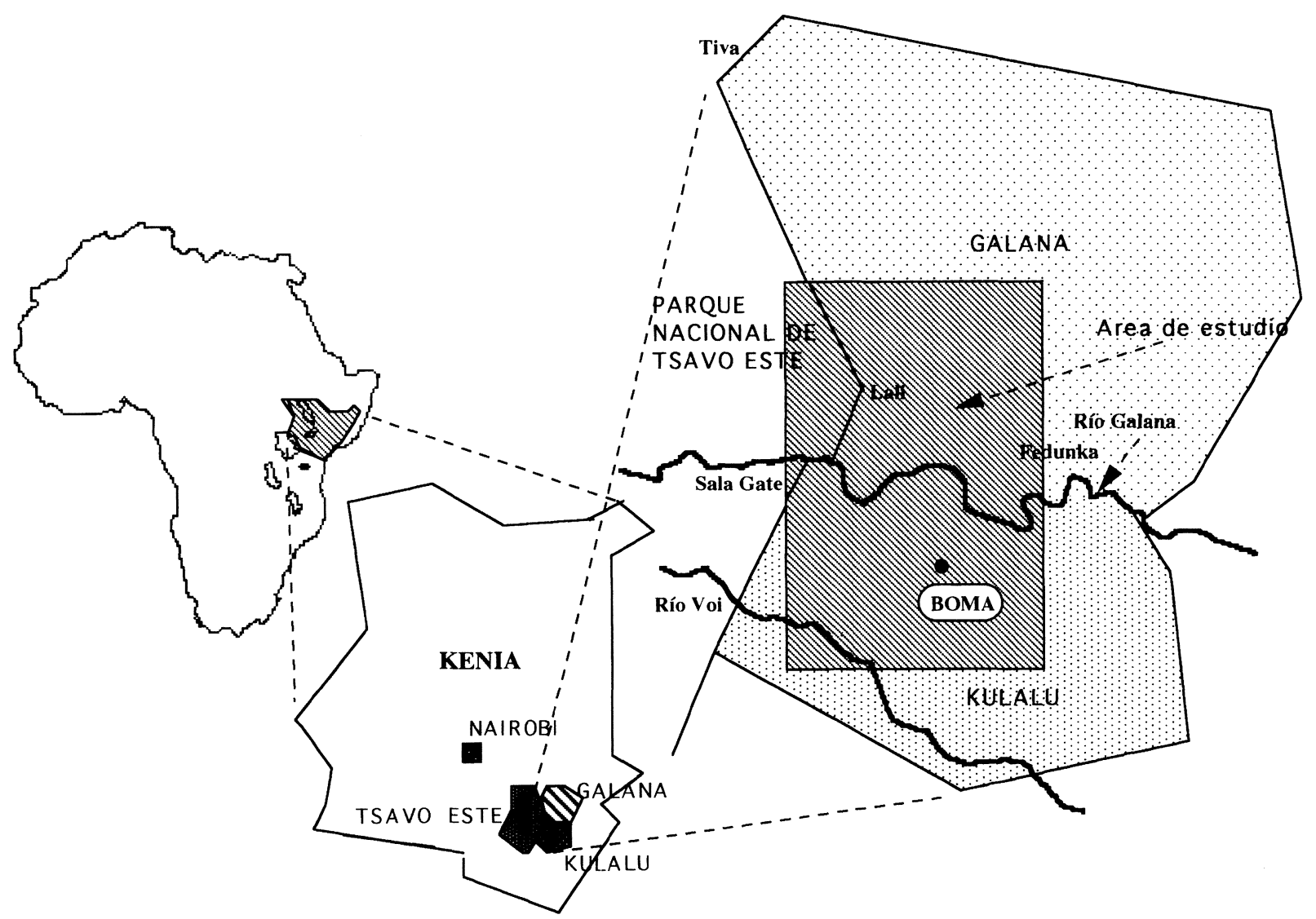

Fig. 1. Localización geográfica del área de estudio y ubicación del campamento (Boma) Maasai.

ña de un nuevo proyecto de investigación etoarqueológica y tafonómica en las reservas faunísticas de Galana, Kulalu y el Parque Nacional de Tsavo Este, en el sureste de Kenia, bajo la dirección y subdirección de los que firmamos este artículo respectivamente (Fig. 1). En el curso de la realización de un estudio en Kulalu sobre la alteración de carcasas por parte de leones y hienas y de la distribución diferencial ósea según el tipo de hábitat, localizamos una Boma (campamento) temporal de pastores nómadas Ndorobo (Maasai), que fue ocupada durante unos pocos días y en la que llevaron a cabo el consumo de una vaca. Juzgamos que era ideal para someter nuestros planteamientos a prueba y después de su abandono, tras un prudente período de exposición de los restos a la acción de los carnívoros carroñeros, decidimos acometer su estudio.

\section{LOS ASENTAMIENTOS NDOROBO (MAASAI) DE GALANA Y KULALU}

Los Maasai comprenden un nutrido grupo de gentes pertenecientes al grupo étnico nilótico, cuya expansión tuvo lugar históricamente desde la franja nororiental del continente africano hasta Tanzania. En la actualidad, se encuentran divididos en tres grandes grupos. El primero de ellos, el Maasai propiamente, abarca un amplio territorio del centro y sur de Kenia y norte de Tanzania. El segundo lo constituyen los Samburu, situados en las altas mesetas del norte de Kenia, entre el monte Kenia y el lago Turkana. El tercero está formado por los Ndorobo (distintos de los Dorobo de las tierras altas), localizados gegráficamente en el sector suroriental de Kenia. Aunque estructuralemente la cultura de todos estos grupos es la 
misma, existen sutiles diferencias, sobre todo en sus conductas económicas y subsistenciales. En el caso de los Ndorobo, que es la etnia Maasai con la que hemos trabajado, una buena parte de sus integrantes, sobre todo los asentados en las zonas de Galana y Kulalu se dedica a la ganadería vacuna como asalariados, siendo controlada su explotación directamente por el gobierno keniata.

Los campamentos de estos Maasai se caracterizan por una organización espacial sencilla, ya que se trata de asentamientos temporales utilizados básicamente durante la estación seca y cuyo período de ocupación no suele exceder los dos meses. Su estructura se compone esencialmente de un cercado para el ganado, al margen del cual se localizan las cabañas y cuyo número suele variar dependiendo del tamaño del grupo. Esta área suele a su vez estar protegida por un cercado de arbustos de menor altura. Todo ello está construido en materiales naturales, a excepción de las cabañas, para las cuales se emplea un material de contrachapado que facilita su construcción y posterior traslado.

La elección del emplazamiento está determinada por dos factores: por un lado, la proximidad a los cursos de agua, y por otra parte, el acceso a buenas zonas de pasto para el ganado. Las actividades que se llevan a cabo en estos campamentos son esencialmente las destinadas al cuidado y mantenimiento del ganado y las relacionadas con la preparación y consumo de alimentos, siendo estas últimas realizadas en el exterior de las viviendas y en torno a los hogares.

La alimentación de estos grupos se basa principalmente en el consumo de harina de maíz (posho), leche, carne vacuna y de pequeñas aves. Cuando descuartizan un animal, lo hacen en una zona periférica del campamento, utilizando para dicho proceso cuchillos y machetes de metal. La primera labor es la extracción de la piel, que posteriormente se extiende para su secado en una zona exterior del campamento o por el contrario, en un espacio cercano a la cabaña.

El animal es desarticulado en su totalidad, transportando prioritariamente las partes pertenecientes al esqueleto axial y apendicular, por este orden. La introducción del cráneo en el campamento no tiene una función subsistencial, ya que sólo se hace para servirse de él como asiento. La carne es asada para su consumo inmediato o, en algunas ocasiones, ahumada para un consumo posterior. Una vez finalizado el contenido cárnico, se pasa a la extracción de la médula ósea, que se consume generalmente sin ningún tipo de preparación previa, excepto el calentamiento ligero de los huesos.

El resultado de todo este proceso crea zonas de acumulación y dispersión de restos bien diferenciadas, que tras el abandono de los asentamientos quedan expuestos a la acción de los animales carroñeros.

\section{ESTUDIO DE LA BOMA MAASAI}

\section{Procedimiento y método}

El campamento temporal que procedimos a estudiar, denominado Boma Di, fue ocupado en el mes de julio, durante tres semanas y media y abandonado a finales de dicho mes, por nueve hombres, de los cuales seis eran Ndorobo, dos Maasais y un Giriama. En ella pernoctaban y comían, manteniendo el ganado pastando durante el día - alejado de la Boma - y en una zona reservada de la misma durante la noche (Fig. 2; Área D). El centro de la Boma, en torno al cual se realizó la mayoría de las actividades, lo constituía un hogar (Fig. 2; Área B). Durante la mayor parte de su ocupación, el grupo de pastores se alimentó de leche, té, alubias y posho (harina de maíz). Sólo en la última semana pudieron consumir una vaca adulta. Tras su abandono, durante todo el mes de agosto y la mitad de septiembre, los restos acumulados estuvieron expuestos a la acción de hienas y chacales.

El 16 de septiembre procedimos a excavar la Boma. En una prospección preliminar observamos que la cantidad de materiales era escasa, siendo la mayor parte de ellos perecederos (palos para encender fuego, para preparar el posho, estacas para asar la carne y cuerdas vegetales) y los únicos restos óseos pertenecían a la mencionada vaca. Inmediatamente,

T. P., 53, n. ${ }^{\circ} 2,1996$ 


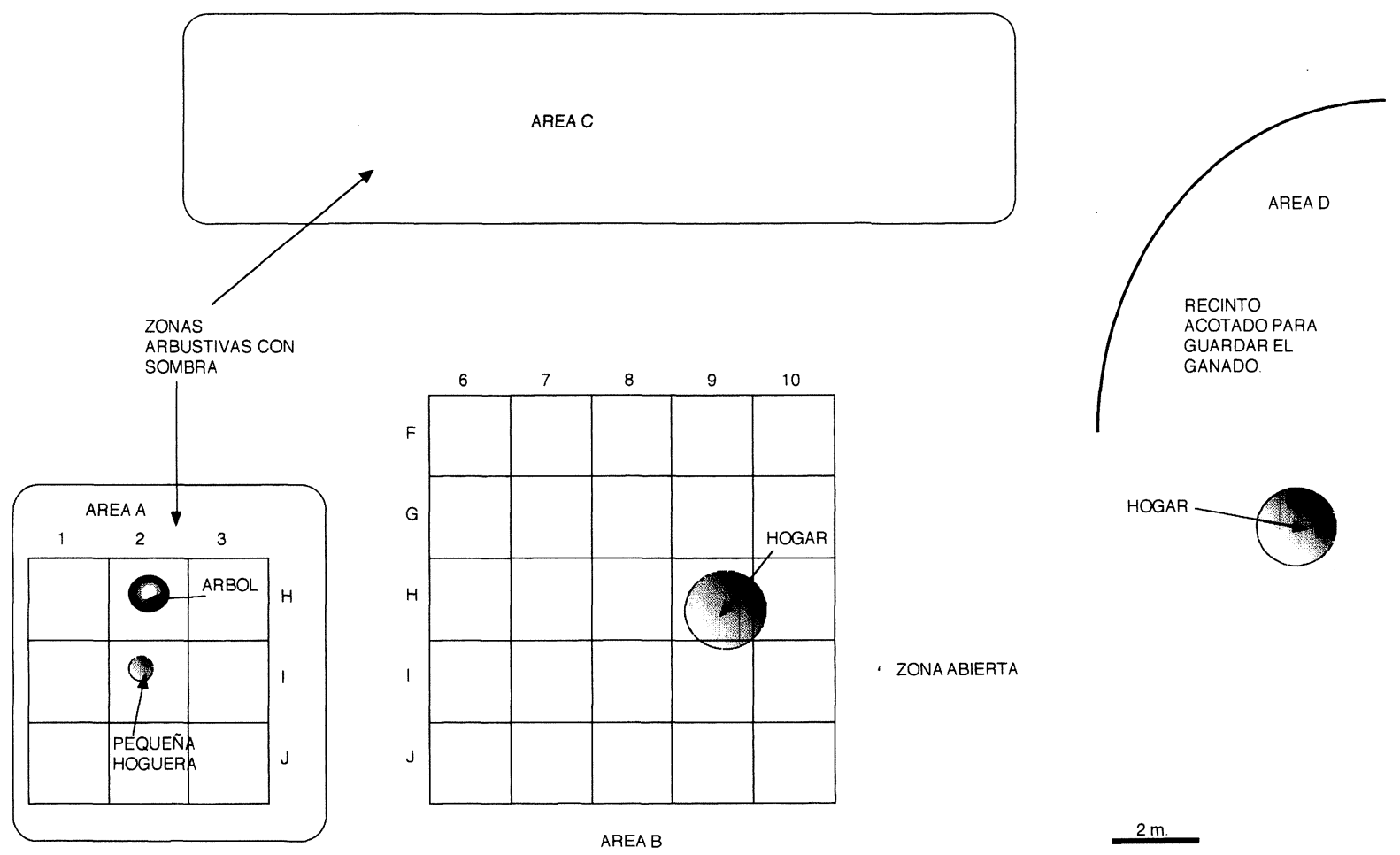

Fig. 2. Plano de distribución de las cuatro áreas principales del campamento, con las dos zonas acotadas con cuadrícula para su excavación.

nos dimos cuenta de que los materiales aparecían concentrados en dos áreas concretas: una, en una explanada despejada, en donde se encontraba el hogar principal (Área B), y otra, a escasa distancia de la anterior (6 m. al este), en torno a un árbol (Área A) (Fig. 2). Entre ambas y en la periferia aparecían elementos aislados y en escaso número, especialmente en una zona umbrosa de arbustos a escasa distancia (Área C). Por ello, decidimos delimitar una zona de $100 \mathrm{~m}^{2}$ en la primera área y de 36 $\mathrm{m}^{2}$ en la segunda (abarcando sendas acumulaciones materiales), en las que establecimos cuadrículas de $2 \times 2 \mathrm{~m}$. Acto seguido, y tras limpiar de matojos la zona acotada, procedimos a documentar en coordenadas la situación de todos los restos y los retiramos. A continuación, excavamos cada cuadrícula, rebajando un nivel superficial de $5 \mathrm{~cm}$. El objeto de esta estrategia era localizar todos los fragmentos pequeños y esquirlas óseas que nos indicasen los enclaves en los que se fracturaron los huesos apendiculares para extraer el tuétano. Debido al posible pisoteo de estos pequeños restos y su probable sedimentación, pensamos que un rebaje de dichas proporciones era necesario y suficiente. El sedimento de cada cuadrícula fue cribado meticulosamente en seco y se recogieron todas las astillas óseas que se observaron, detectándose hasta esquirlas menores de $1 \mathrm{~cm}$.

Además de las zonas delimitadas, posteriormente se procedió a prospectar y recoger los materiales dispersos en un radio de $250 \mathrm{~m}$. entorno al área B. El objetivo era recuperar posibles fragmentos y piezas que los carnívoros suelen dispersar tras su acceso secundario.

Luego, el material fue examinado de manera preliminar en el laboratorio de campo, y con más minuciosidad en el laboratorio de Prehistoria de la Universidad Complutense de Madrid. Allí cada resto se escrutinó con lupa binocular de $20 \mathrm{X}-80 \mathrm{X}$ para asegurarse de la identidad de cada tipo de marca detectada (marcas de corte, dientes, percusión...). También se realizaron remontajes de piezas. 


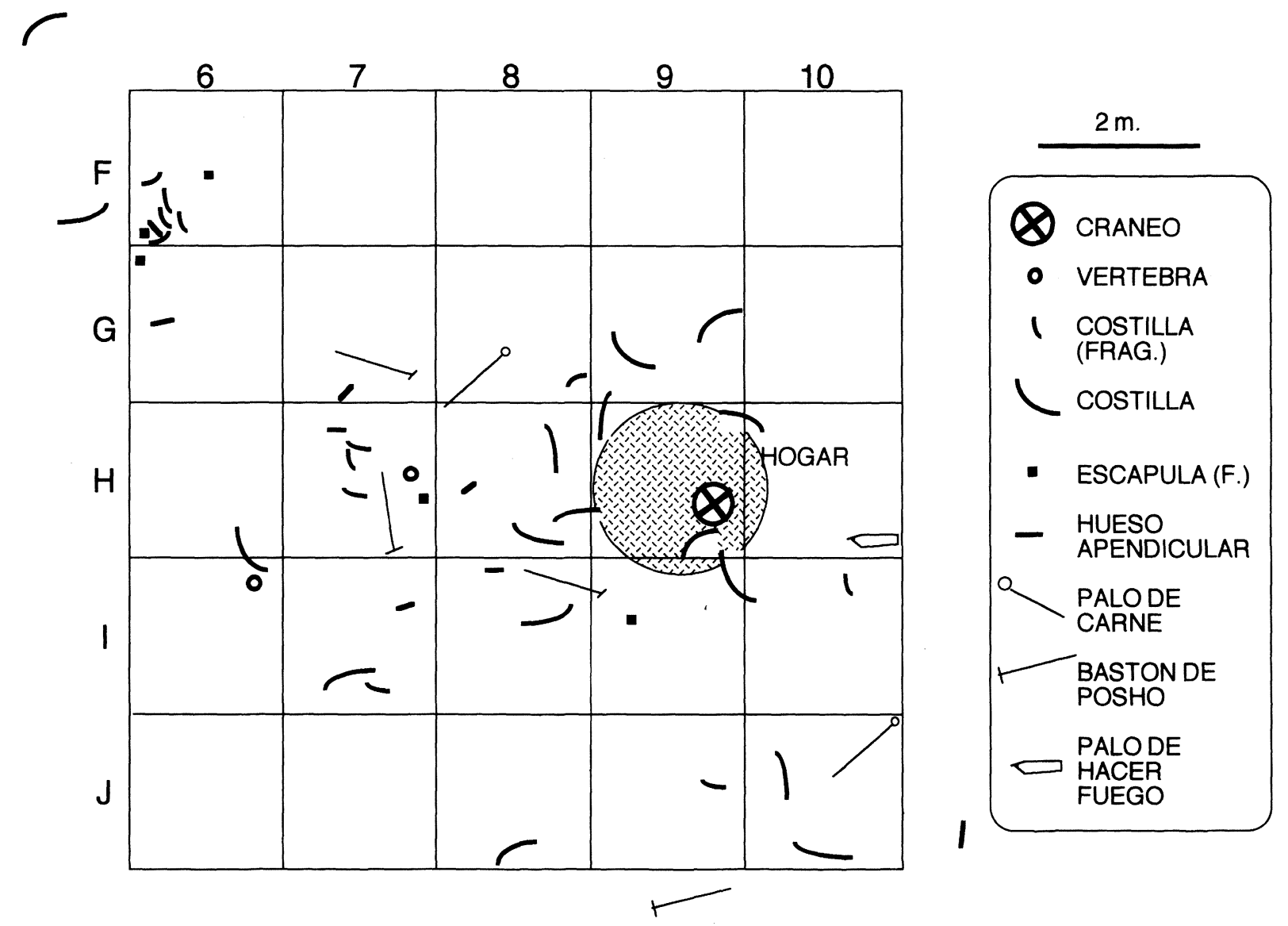

Fig. 3. Plano de distribución espacial de los materiales existentes en el área B.

\section{Distribución de los restos óseos}

Dentro de las cuatro áreas en las que se observaron evidencias de ocupación -el árbol al oeste (Área A), la zona despejada (Área B), la zona septentrional arbustiva (Área C) y la zona oriental para guardar el ganado (Área D) (Fig. 2 ) - , los restos óseos se restringen en más de un $90 \%$ a las dos primeras y la mayoría se encuentra en el área $\mathrm{B}$, caracterizada por un gran hogar. En el área $C$ aparecen algunos huesos dispersos y aislados, mientras que en el área $\mathrm{D}$ están ausentes.

Si analizamos el área $\mathrm{B}$, nos encontramos con una distribución espacial de restos significativa (Fig. 3). En el entorno inmediato del hogar aparecen palos de carne (usados para clavar trozos y mantenerlos al calor del hogar sin que el fuego los alcance) y bastones de posho (usados para remover la harina de maíz mientras se cocina). También aparecen huesos que, en los $36 \mathrm{~m}^{2}$ que rodean al hogar -y situándose éste como punto central (cuadrículas G8-G10, H8-H10, I8-I10) - son, salvo dos fragmentos apendiculares, pequeños restos de costillas y costillas más o menos completas. Los huesos de las extremidades aparecen a mayor distancia (cuadrículas G7, H7, I7 y G6). Esta distribución es el resultado de la conducta habitual de estas poblaciones al preparar la carne al fuego y consumirla en torno al mismo, siendo los elementos que sólo contienen carne procesados y descartados de inmediato alrededor del hogar. Precisamente, los huesos axiales, como elementos de estas características, son los que se abandonan en semejante lugar, tras un único 
acto de consumo, tal y como puede observarse en la figura 3. Los huesos apendiculares, sometidos al mismo proceso, puesto que se seccionan y se clavan en los palos de carne para prepararlos al fuego, sufren una manipulación antrópica dividida en dos fases: la primera, orientada al consumo de la carne, y la segunda, con el objeto de extraer su médula. Una buena parte de la primera fase - si no toda, dependiendo de la ocasión - se realiza en torno al fuego. Sin embargo, a veces, la segunda implica un desplazamiento de los huesos descarnados a otro sitio, en el que son fracturados. El consumo del tuétano puede realizarse allí o en un tercer lugar. Esta actitud podría explicar que los huesos apendiculares del área B aparezcan más alejados del hogar que las costillas. Para conocer con mayor precisión si el lugar en el que aparecen fue el sitio en el que también fueron fracturados y de consumo cárnico/medular o sólo consumo cárnico, es preciso cotejar la información espacial de los macrorrestos con la de los microrrestos (astillas y esquirlas óseas), como haremos posteriormente.

También llama la atención la presencia de elementos escapulares fragmentados, que atestiguan parte de la desarticulación del animal en dicha área. Sabemos que el proceso completo de matanza, descuartizamiento y parte del consumo de la vaca tuvo lugar en la misma, por el hecho de que la mayor parte de los huesos especialmente los axiales - se encuentra allí, junto con el cráneo, y porque en la cuadrícula J8 recuperamos un pequeño palo típico, seccionado en dos partes, utilizado para clavarlo al suelo y atar al animal para poder matarlo.

En claro contraste con esta situación, la mayor parte de los huesos desplazados al área A, probablemente buscando la sombra del árbol allí existente, es de carácter apendicular: ocho de los nueve huesos recuperados dentro de la zona acotada pertenecen a las extremidades (Fig. 4). Normalmente, estos huesos se acercan al fuego cuando aún contienen carne y su preparación no deja ninguna alteración térmica sobre su superficie. Una vez que se extrae la carne, algunos de los huesos suelen someterse una vez más al fuego para calentar la médula previamente a su fractura e ingesta. En este proceso síllegan a quemarse determinadas sec-

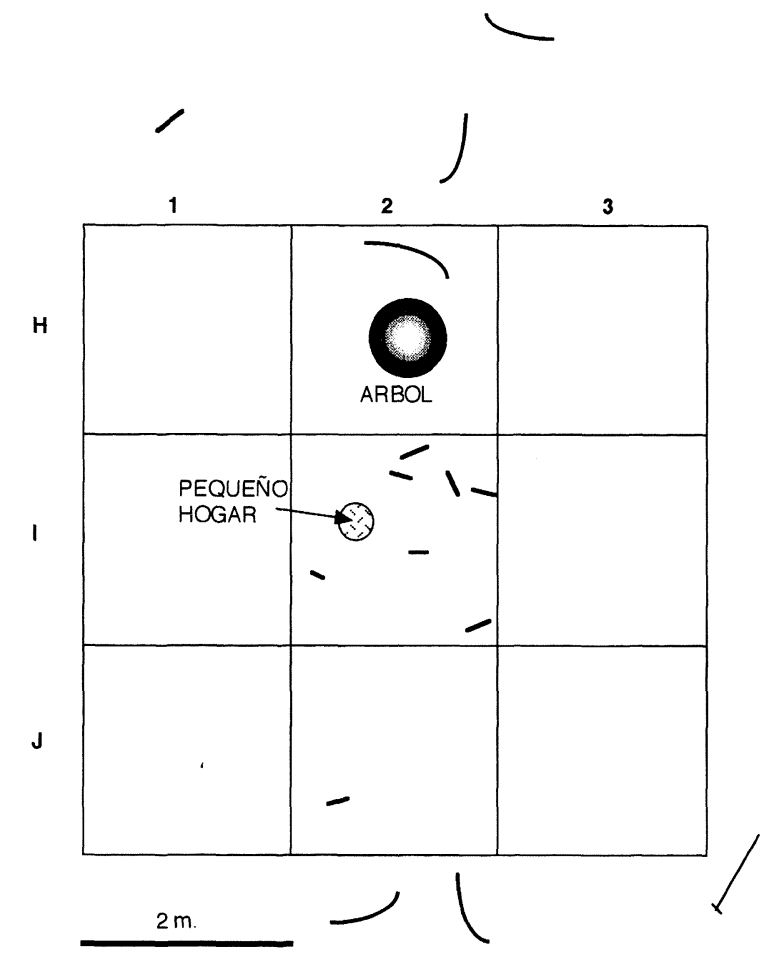

Fig. 4. Plano de distribución espacial de los materiales existentes en el área A (misma clave que en figura 3).

ciones de los mismos. La presencia de un pequeño hogar en proximidad al árbol, insuficiente para calentar la carne, pero de dimensiones apropiadas para calentar huesos sueltos, indicaría que en dicho sitio se consumió el tuétano de algunos de ellos. El análisis de la distribución espacial de las astillas y esquirlas óseas dará mayor apoyo a dicha interpretación.

Fuera de las zonas cuadriculadas aparecen huesos dispersos y en menor cantidad, cerca de las mismas y en el área $\mathrm{C}$, también arbustiva y umbrosa como el área $\mathrm{A}$.

\section{Distribución espacial de las astillas óseas}

Con el fin de conocer los lugares en los que se fracturaron los huesos para extraer el tuétano, la excavación superficial de las áreas acotadas y el cribado de sedimento permitió recuperar un total de 113 astillas y esquirlas óseas, la mayor parte $(77 \%)$ menores de $2 \mathrm{~cm}$. y el resto 
entre 2 y $8 \mathrm{~cm}$. Ante nuestra sorpresa nos encontramos - salvo el cuadro $\mathrm{H} 10$, en el que sólo apareció un fragmento- con que los restos de estas características aparecían muy concentrados y en cantidades significativas dentro de ciertas cuadrículas, siendo inexistentes en el resto. $\mathrm{Cu}$ riosamente, la primera agrupación aparece en el área $\mathrm{B}$, en torno al hogar central. De hecho, el $64 \%$ de los restos de fractura - $6 \%$ más si contamos los recuperados en el cuadro F6 - se encuentran cerca del fuego, algo más alejados que los huesos axiales y junto con los macrorrestos pertenecientes a las extremidades (Fig. 5).

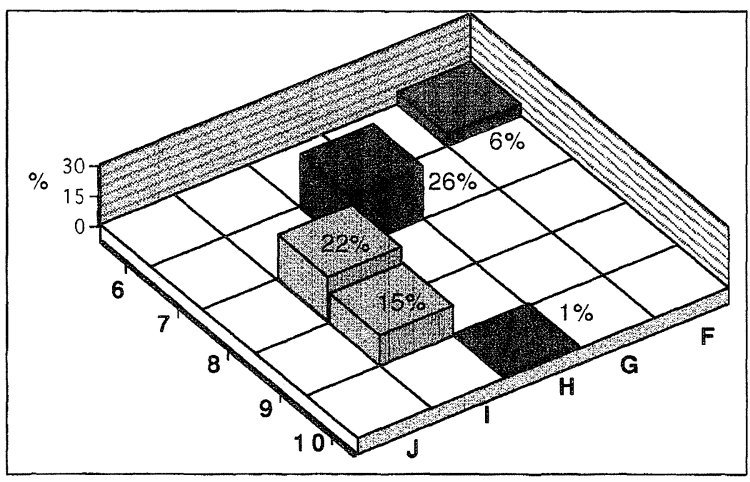

Fig. 5. Distribución porcentual y espacial de las astillas y pequeñas esquierlas óseas recuperadas en el área B, tras su excavación.

La otra agrupación aparece en al área A, alrededor del pequeño hogar, y en conexión con los huesos apendiculares de mayor tamaño. El $30 \%$ de los fragmentos de fractura localizados aparece allí (Fig. 6).

Esto indica la existencia de dos zonas claras de extracción de médula, ambas en torno al fuego y una de ellas - la del área A- explicable por el desplazamiento de restos a la misma debido probablemente a que dispensaba sombra.

Este estudio confirma la presencia de dos áreas diferenciadas de actividad subsistencial en función del producto (carne/tuétano) consumido: el área $\mathrm{B}$, en donde se mata y descuartiza al animal, se prepara y consume su carne, con cierto grado de ingesta de médula, y el área $\mathrm{A}$, a donde se desplazan ciertos huesos descarnados para su aprovechamiento medular exclusi-

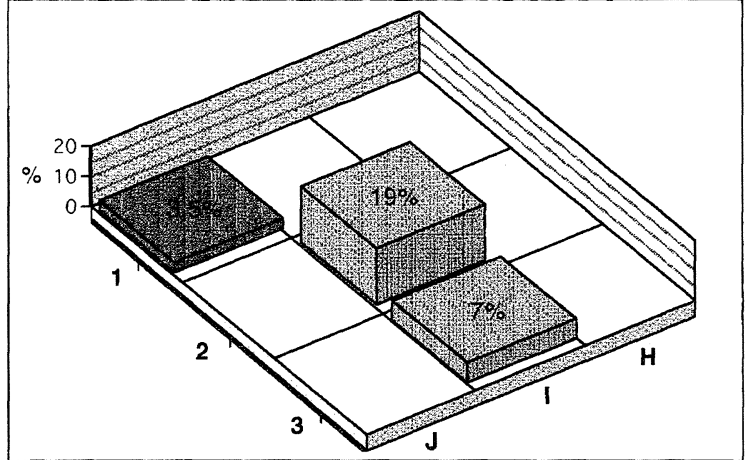

Fig. 6. Distribución porcentual y espacial de las astillas y pequeñas esquirlas óseas recuperadas en el área $\mathrm{A}$, tras su excavación.

vamente (salvo que se hubiese consumido su carne también allí, aunque hubiese sido preparada anteriormente en el hogar del área $\mathrm{B}$ ).

\section{Remontajes de piezas óseas}

$\mathrm{El}$ intento de encontrar piezas que encajasen resultó arduo y con escasos frutos, dado el carácter de la alteración posdeposicional a la que se vio sometido todo el conjunto óseo. No obstante, fuera de los recintos acotados encontramos en el espacio situado entre las áreas A, $\mathrm{B}$ y $\mathrm{C}$ dos restos apendiculares que encajaban, estando situados a bastante distancia el uno del otro. Probablemente, los carnívoros carroñeros fueron los responsables de semejante dispersión, al ser ambos huesos de tipo epifisario. Sin embargo, en el área B pudimos remontar tres piezas, pertenecientes a un húmero, que se encontraba cada una en cuadros diferentes (G7, H7 e I7). Con ello documentábamos que dicha zona, en la que el análisis de distribución de esquirlas óseas demostraba una actividad de fractura de huesos, también fue enclave de consumo de médula.

\section{Marcas de corte}

Una gran cantidad de huesos posee marcas de corte producidas durante el procesamiento y consumo de la vaca (Tabla 1). Éstas se encuen- 


\begin{tabular}{|l|c|c|c|c|}
\hline & $\begin{array}{c}\text { Número } \\
\text { total } \\
\text { de } \\
\text { piezas }\end{array}$ & $\begin{array}{c}\text { Número } \\
\text { de huesos } \\
\text { con marcas } \\
\text { de corte }\end{array}$ & $\begin{array}{c}\% \text { de piezas } \\
\text { de esta parte }\end{array}$ & $\begin{array}{c}\% \text { de } \\
\text { todas las } \\
\text { piezas con } \\
\text { marcas }\end{array}$ \\
\hline CRÁNEO & 1 & - & - & - \\
MAND. & - & - & - & - \\
VÉRT. & 5 & 3 & 60 & 7,3 \\
COST. & 41 & 21 & 51 & 51,2 \\
ESC. & 6 & 3 & 50 & 7,3 \\
PELVIS & 1 & 1 & 100 & 2,4 \\
HÚM. DP & 1 & - & - & - \\
HÚM.D & 5 & 4 & 80 & 9,7 \\
HÚM. DD & 4 & 4 & 100 & 9,7 \\
RAD. DD & 3 & 1 & 33,3 & 2,4 \\
RAD. D & 2 & 1 & 50 & 2,4 \\
RAD. DD & 1 & - & - & - \\
MC. DP & 1 & - & - & - \\
MC. D & - & - & - & - \\
MC. DD & - & - & - & - \\
FÉM. DP & 1 & - & - & - \\
FÉM. D & 3 & 2 & 66,6 & 4,8 \\
FÉM. DD & - & - & - & - \\
TIB. DP & 1 & - & - & - \\
TIB.D & - & - & - & - \\
TIB. DD & 2 & 1 & 50 & 2,4 \\
MT. DP & - & - & - & - \\
MT.D & - & - & - & - \\
MT. DD & - & - & - & - \\
ASTRÁGALO & - & - & - & - \\
CALCÁNEO & - & - & - & - \\
\hline TOTAL & $\mathbf{8 0}$ & $\mathbf{4 1}$ & & $\mathbf{1 0 0}$ \\
\hline
\end{tabular}

Tabla 1. Representación del número y tipo de restos y correspondientes marcas de corte. Clave: MAND: mandíbula; VERT: vértebra; ESC.: escápula; HÚM.: húmero; RAD.: radio; MC.: metacarpo; FÉM.: fémur; TIB.: tibia; MT.: metatarso; DP.: diáfisis proximal; D.: diáfisis central; DD.: diáfisis distal.

tran distribuidas, en términos porcentuales, de la siguiente manera:

- La mitad de los especímenes óseos (51\%) con marcas de corte pertenecen a las costillas.

- Las vértebras $(7 \%)$, escápulas $(7 \%)$ y pelvis $(2 \%)$ también cuentan con este tipo de alteración.

- Con respecto a las extremidades, el húmero $(19 \%)$ y radio $(5 \%)$ aglutinan el mayor porcentaje de marcas, mientras que el fémur $(5 \%)$ y tibia $(2 \%)$ poseen un índice menor, debido a su peor conservación.

Si observamos la representación de estas macas en función del número de piezas del mismo tipo, los porcentajes son distintos. Las

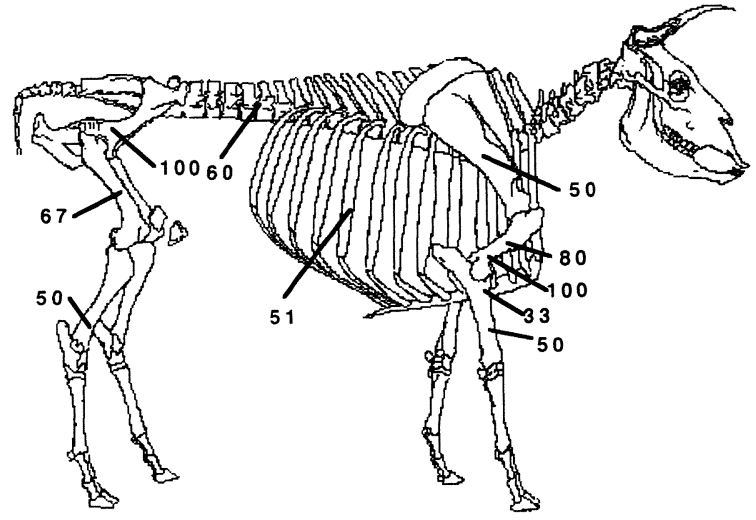

Fig. 7. Distribución porcentual y anatómica de las marcas de corte en los restos de la vaca.

costillas siguen manteniendo un índice superior al $50 \%$ de representación, mientras que las vértebras aumentan al $60 \%$, la escápula al $50 \%$ y la pelvis al $100 \%$. La sección distal del húmero también alcanza el $100 \%$ y su diáfisis central el $80 \%$, algo más reducidos en el radio $-33 \%$ la parte proximal y $50 \%$ la diáfisis central- . El resto de porcentajes aparece en la diáfisis del fémur $(67 \%)$ y la sección distal de la tibia (50\%) (Tabla 1, Fig. 7).

Las marcas, que aparecen aisladamente o en grupos, se encuentran distribuídas de la siguiente manera:

- Costillas: aparecen en varias partes de las mismas. Son frecuentes en la sección media (por ambas caras del hueso) y también en su sección proximal.

- Vértebras: aparecen a lo largo de las apófisis y cerca del cuerpo óseo.

- Extremidades: son mayoritarias en las secciones centrales de las diáfisis, aunque abundan también en las secciones diafisarias proximales y distales (Tabla 1).

\section{ALTERACIONES POSDEPOSICIONALES}

Tras el abandono del campamento, los restos depositados quedaron expuestos durante un mes y medio a la acción de los carnívoros carroñeros (chacales y hienas) que influyeron de dos maneras en la configuración difinitiva del conjunto óseo analizado: la alteración de un 


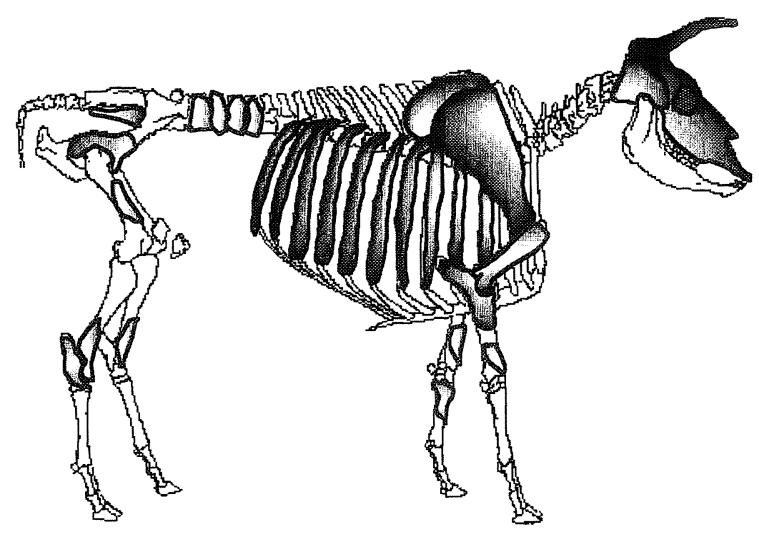

Fig. 8. Representación de los restos óseos conservados en el campamento Maasi estudiado (las zonas oscuras indican presencia y las claras ausencia).

amplio número de piezas y la desaparición de otras por consumo y/o desplazamiento.

\section{Representación esquelética}

Las piezas presentes en el conjunto óseo son: el cráneo completo, la práctica totalidad de las costillas, cinco vértebras, un fragmento del acetábulo pélvico, una escápula casi completa y varios fragmentos de la otra, dos húmeros, dos radios, una ulna, un fémur, dos tibias, un astrágalo, un calcáneo y un metápodo. Las secciones óseas de los huesos apendiculares son: un fragmento de epífisis proximal y las dos epífisis distales de húmero, un fragmento de la epífisis proximal de radio, un fragmento de la sección distal de la ulna, las dos epífisis distales de las tibias y una epífisis proximal del metacarpo. El resto de las piezas pertenecientes a las extremidades, que constituyen la mayor parte, son fragmentos diafisarios de la parte proximal/distal o central de cada elemento (Fig. 8). La ausencia de mandíbula, de la mayoría de las vértebras y la pelvis, así como de de las regiones epifisarias - especialmente, las de menor densidad (proximal de húmero y tibia, las distales de radio y las femorales) - son indicativos de una intensa acción destructiva de las hienas. Del mismo modo, la práctica ausencia de metápodos y huesos distales pequeños (tarsales, carpales, falanges...) indica un transporte y dispersión por parte de estos agentes $\mathrm{y} / \mathrm{o}$ chacales.

\begin{tabular}{|l|c|c|c|c|}
\hline & $\begin{array}{c}\text { Número } \\
\text { total } \\
\text { de } \\
\text { piezas }\end{array}$ & $\begin{array}{c}\text { Número } \\
\text { de huesos } \\
\text { con marcas } \\
\text { de diente }\end{array}$ & $\begin{array}{l}\% \text { de piezas } \\
\text { de esta parte }\end{array}$ & $\begin{array}{c}\% \text { de } \\
\text { todas las } \\
\text { piezas con } \\
\text { marcas }\end{array}$ \\
\hline CRANEO & 1 & - & - & - \\
MAND. & - & - & - & - \\
VERT. & 5 & 2 & 40 & 8 \\
COST & 41 & 18 & 44 & 72 \\
ESC & 6 & 2 & 33 & 7,3 \\
PELVIS & 1 & 1 & 100 & 8 \\
HUM DP & 1 & - & - & - \\
HUM D & 5 & 1 & 20 & 4 \\
HUM DD & 4 & 1 & 25 & 4 \\
RAD DD & 3 & - & - & - \\
RAD D & 2 & - & - & - \\
RAD DD & 1 & - & - & - \\
MC DP & 1 & - & - & - \\
MCD & - & - & - & - \\
MC DD & - & - & - & - \\
FEM DP & 1 & - & - & - \\
FEM D & 3 & 1 & 33 & 4 \\
FEM DD & - & - & - & - \\
TIB DP & 1 & - & - & - \\
TIB D & - & - & - & - \\
TIB DD & 2 & - & - & - \\
MT DP & - & - & - & - \\
MT D & - & - & - & - \\
MT DD & - & - & - & - \\
ASTRAGALO & - & - & - & - \\
CALCANEO & - & - & - & - \\
\hline TOTAL & $\mathbf{8 0}$ & $\mathbf{2 5}$ & & $\mathbf{1 0 0}$ \\
\hline
\end{tabular}

Tabla 2. Representación del número y tipo de restos y correspondientes marcas de diente. Clave: MAND: mandíbula; VÉRT: vértebra; ESC.: escápula; HÚM.: húmero; RAD.: radio; MC.: metacarpo; FÉM.: fémur; TIB.: tibia; MT.: metatarso; DP.: diáfisis proximal.; D.: diáfisis central; DD.: diáfisis distal.

La sobrerrepresentación de costillas (dada su categoría de conservación ateniéndose a los criterios de densidad ósea) en comparación con el resto de elementos axiales se explica por su estado de alteración antrópica. Al poseer una capa cárnica de escaso grosor y ser expuestas al fuego, su abandono se produce tras haber consumido la carne, quedando el hueso quemado. Esto resta atractivo para los carroñeros que, a pesar de ello, las modificaron de manera significativa como veremos a continuación.

\section{Marcas de dientes}

El índice de huesos con marcas de dientes es sumamente elevado en comparación con otros 
conjuntos antrópicos en los que los carroñeros intervienen de manera secundaria: 25 de los 80 huesos que forman parte de la acumulación de la Boma $(31 \%)$ tienen al menos una marca de diente (Tabla 2). El 72\% de todos los especímenes con marcas de este tipo lo constituyen costillas, seguidas por vértebras $(8 \%)$ y escápulas (8\%). El $12 \%$ restante lo forman huesos apendiculares: solamente 3 especímenes (un epífisis distal de húmero y dos fragmentos diafisarios de estilopodios).

El tipo de marca más frecuente suele ser la fosa superficial (pit), casi exclusiva en los huesos apendiculares, junto con algunos surcos (scores) poco definidos. En las costillas y vértebras abundan las perforaciones (punctures) y las fosas (pits) más profundas. En las costillas suelen aparecer en las secciones distales, siendo menos frecuentes en las secciones proximales. El 44\% de estos huesos tiene alguna marca de diente.

\section{Dispersión de huesos}

Ya hemos comentado anteriormente que la mayoría de los huesos aparece en dos concentraciones de origen antrópico, en sendas áreas A y B. Sin embargo, observamos que 16 de los 80 huesos recuperados $(20 \%)$ se recogieron fuera de las áreas acotadas. Cinco de los siete huesos en torno al área A se encontraban dentro de un radio inferior a los $6 \mathrm{~m}$. y tres de los ocho huesos recogidos cerca del área $\mathrm{B}$ estaban a similar distancia. Los otros (ocho especímenes) estaban más alejados (Fig. 9). Sin embargo, la mayoría estaba en el área C (Fig. 2), más umbrosa y podría deberse a aporte tanto humano, como carnívoro. El hecho de que cinco de ellos fuesen costillas, y que todos tuviesen marcas de dientes señalan a un agente carnívoro como responsable principal. Los huesos encontrados cerca de las áreas $\mathrm{A}$ y $\mathrm{B}$ podrían deber su presencia allí por haber sido arrojados desde las zonas de consumo. Los más alejados deben su situación, presumiblemente, a la acción de hienas y chacales. El hecho más extremo que pudimos documentar de la intervención de estos agentes fue el descubrimiento de una tibia distal y un fragmento de metápodo a 200 m. del centro del área B (Fig. 9).

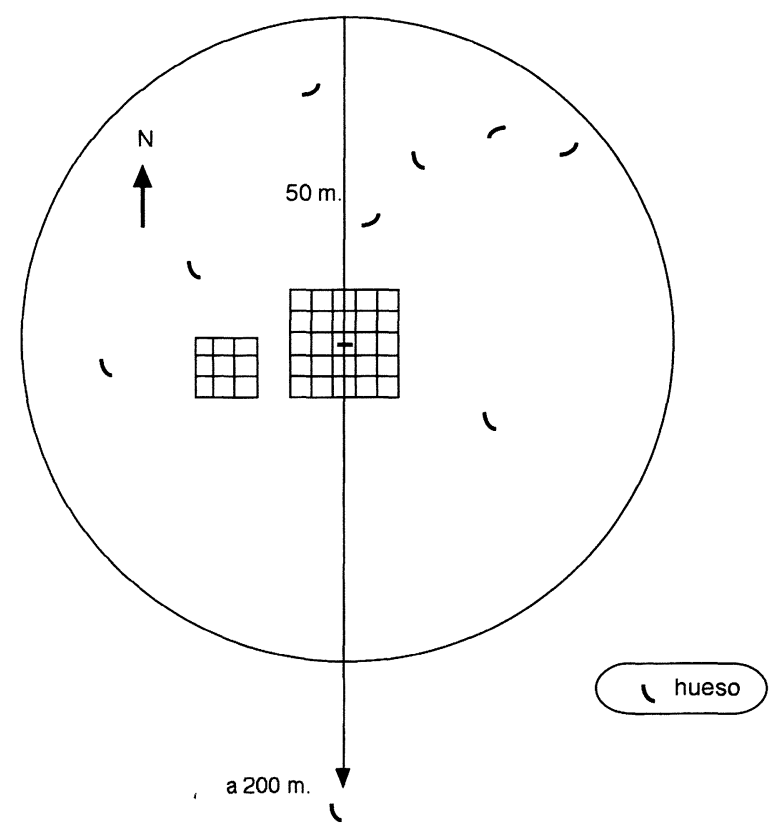

Fig. 9. Patrón de distribución espacial de restos en la periferia de las zonas acotadas.

\section{DISCUSIÓN}

El estudio de la Boma Ndorobo aporta una serie de elementos para la reflexión sobre el estudio de los suelos de ocupación arqueológicos.

1. Un episodio de ocupación tan breve y en el que se llevó a cabo una actividad tan puntual como fue el consumo de una vaca, ha dejado evidencia material en un área muy amplia. Si sólo quisiésemos abarcar mediante estrategia arqueológica las dos acumulaciones antrópicas principales, habría sido necesario acotar una zona de 10 × 5 cuadrículas (de $2 \times 2$ m.) en un total de $200 \mathrm{~m}^{2}$. Si deseásemos incluir la diseminación periférica de restos, se habría hecho necesario triplicar dicha extensión (excluyendo las piezas más alejadas). Esas dimensiones son similares (aunque ligeramente inferiores) a las documentadas por Gifford (1977) en los campamentos temporales de las poblaciones Dassanetch en el lago Turkana (Kenia) y a las de los bosquimanos (Bartram et alii, 1991).

2. El estudio del grado (cantidad) y carácter (tipo de hueso) de las acumulaciones y su dis- 
tribución espacial resulta indicativo del lugar de matanza y consumo de una carcasa.

3. El análisis de la distribución espacial de los pequeños fragmentos óseos indica los lugares en los que se llevó a cabo la ruptura de huesos para extraer y consumir el tuétano.

4. Los índices de representación de marcas de corte y su distribución anatómica indican el tipo de aprovechamiento cárnico obtenido del animal. Una frecuencia elevada de marcas en los elementos de mayor valor cárnico - huesos axiales y apendiculares superiores (diáfisis) sugiere su explotación primaria.

5. La destrucción ósea debida a la intervención secundaria de carnívoros carroñeros se centra en los elementos axiales. A este respecto, la acumulación estudiada coincide con la experimentación realizada sobre la actuación de hienas en conjuntos antrópicos (Marean et alii, 1992). En el caso de la Boma, la elevada supervivencia de las costillas se debe a que una buena parte de ellas sufrió el efecto del fuego, mermando de esta manera el interés por parte de los carnívoros. Aun así, el $44 \%$ de ellas fue alterado por estos agentes y muchas desplazadas de su lugar de deposición original. La destrucción de epífisis con respecto a las diáfisis es evidente y el índice entre ambas secciones óseas resulta indicativo del acceso secundario de los carroñeros (Blumenschine, 1988; Blumenschine y Marean, 1993; Capaldo, 1995). Es interesante señalar la desaparición de las partes distales de las extremidades, transportadas del lugar.

6. El porcentaje de huesos con marcas de dientes es muy elevado ( $31 \%$ ), situándose en la parte alta del espectro obtenido en la experimentación con conjuntos antrópicos alterados por hienas (Blumenschine, 1988). Semejante índice se debe a la alta incidencia de marcas de dientes en los elementos axiales y a la elevada (y anómala) supervivencia de costillas. Si sólo nos fijamos en las extremidades, el porcentaje de este tipo de alteración se reduce al $12 \%$, similar al término medio obtenido mediante la experimentación aludida (Blumenschine, 1988; Blumenschine \& Marean, 1993).

7. Un número reducido, pero significativo (dos especímenes), de fragmentos diafisarios muestran marcas de dientes. Este hecho contradice la aseveración de Blumenschine (1988) de que este tipo de secciones óseas queda prácticamente exento de alteraciones secundarias debidas a carnívoros, por no resultar de interés alimenticio.

En este estudio hemos discernido los resultados materiales de una serie de procesos que han operado sobre un solo animal, en un período de ocupación corto en un mismo enclave. Desgraciadamente, la lectura de los procesos causantes de los yacimientos arqueológicos no resulta tan sencilla por presentar una resolución e integridad menores. Por ello, a partir de este primer paso, nuestra investigación, actualmente en curso, se encamina al análisis de asentamientos ocupados de una manera más prolongada, en los que el número de actividades realizadas es mayor y en los que se han consumido varios animales. De su estudio y cotejo con el asentamiento que hemos presentado en este trabajo esperamos poder definir diagnosis más seguras de aplicación al registro arqueológico para poder ayudar en su inteligibilidad y comprensión.

\section{AGRADECIMIENTOS}

Deseamos expresar nuestro sincero agradecimiento a la Oficina del Presidente, al K.W.S. (Kenya Wildlife Service), y al A.D.C. (Agricultural Development Corporation) de Kenia, por su ayuda y concesión de permisos para llevar a cabo nuestra investigación. Estamos especialmente agradecidos a John J. Anière por su inestimable ayuda de campo y a Mr. Gishangi, director de K.W.S. en Tsavo, por su apoyo y el acceso facilitado al centro de investigación de Tsavo. Este estudio fue realizado gracias a la financiación obtenida a través de un proyecto precompetitivo otorgado por la Universidad Complutense.

\section{BIBLIOGRAFÍA}

Bartram, L.E.; Kroll, E.M. \& BunN, H.T. (1991): "Variability in camp structure and bone food refuse patterning at Kua San hunter-gatherer camps". En E.M. Kroll \& D. Price (ed.): "The interpretation of archaeological spatial patterning”, Plenum Press. New York.

T. P., 53, n. ${ }^{\circ} 2,1996$ 
BINFORD, L.R. (1981): “Bones: ancient men and modern myths". Academic Press. New York.

BLUMENSCHINE, R.J. (1988): “An experimental model of the timing of hominid and carnivore influence on archaeological bone assemblages". Journal of Archaeological Science, 15: 483-502.

BlumensChine, R.J. \& MAREAN, C.W. (1993): "A carnivore's view of archaeological bone assemblages". En: From bones to behavior: Ethnoarchaeological and experimental contributions to the interpretations of faunal remains. J. Hudson (ed.). Southern Illinois University.

BRAIN, C.K. (1981): The Hunters or the Hunted? University of Chicago Press. Chicago.

BunN, H.T. (1982): Meat-eating in human evolution: studies on the diet and subsistence patterns of Plio-Pleistocene hominids in East Africa. Tesis doctoral. Department of Anthropology. University of California. Berkeley.
CAPALDO, S.D. (1995): Inferring hominid and carnivore behavior from dual-patterned archaeofaunal assemblages. Tesis doctoral. Rutgers University. New Jersey.

DomíngueZ-RodRIGO, M. (1994): "Dinámicas tróficas, estrategias de consumo y alteraciones óseas en la sabana africana: resumen de un proyecto de investigación etoarqueológico (1991-1993)". Trabajos de Prehistoria, 51 (1): $15-37$

GIFFORD, D. (1977): Observations of modern human settlements as an aid to archaeological interpretations. Tesis doctoral. Universidad de California. Berkeley.

Marean, C.W.; Spencer, L.M.; Blumenschine, R.J.; CAPAldo, S.D. (1992): "Captive hyaena bone choice and destruction, the Schlepp effect and Olduvai archaeofaunas". Journal of Archaeological Science, 19: 101-121. 